\title{
ARTICLE
}

Clinical Study

\section{A Phase I study of the novel immunomodulatory agent PG545 (pixatimod) in subjects with advanced solid tumours}

\author{
Keith Dredge ${ }^{1}$, Todd V. Brennan², Edward Hammond', Jason D. Lickliter ${ }^{3}$, Liwen Lin², Darryn Bampton', Paul Handley, \\ Fleur Lankesheer ${ }^{4,9}$, Glynn Morrish ${ }^{5}$, Yiping Yang ${ }^{6}$, Michael P. Brown ${ }^{7}$ and Michael Millward ${ }^{8}$
}

\begin{abstract}
BACKGROUND: PG545 (pixatimod) is a novel immunomodulatory agent, which has been demonstrated to stimulate innate immune responses against tumours in preclinical cancer models.

METHODS: This Phase I study investigated the safety, tolerability, pharmacokinetics, pharmacodynamics and preliminary efficacy of PG545 monotherapy. Escalating doses of PG545 were administered to patients with advanced solid malignancies as a weekly 1-h intravenous infusion.

RESULTS: Twenty-three subjects were enrolled across four cohorts (25, 50, 100 and $150 \mathrm{mg})$. Three dose-limiting toxicities (DLTs) hypertension (2), epistaxis (1)-occurred in the $150 \mathrm{mg}$ cohort. No DLTs were noted in the $100 \mathrm{mg}$ cohort, which was identified as the maximum-tolerated dose. No objective responses were reported. Best response was stable disease up to 24 weeks, with the disease control rate in evaluable subjects of $38 \%$. Exposure was proportional up to $100 \mathrm{mg}$ and mean half-life was $141 \mathrm{~h}$. The pharmacodynamic data revealed increases in innate immune cell activation, plasma IFN $\gamma$, TNFa, IP-10 and MCP-1.

CONCLUSION: PG545 demonstrated a tolerable safety profile, proportional PK, evidence of immune cell stimulation and disease control in some subjects. Taken together, these data support the proposed mechanism of action, which represents a promising approach for use in combination with existing therapies.
\end{abstract}

British Journal of Cancer https://doi.org/10.1038/s41416-018-0006-0

\section{INTRODUCTION}

Immunotherapy is an effective treatment option for cancer patients across a broad range of tumour types. The durable and long-lasting anti-tumour activity observed in patients responding to immune checkpoint inhibitors have propelled multiple agents into mainstream cancer therapy. ${ }^{1}$ Nevertheless, most patients do not respond ${ }^{2}$ and intrinsic resistance is common, ${ }^{3}$ so finding therapeutic combinations that convert nonresponders to responders is critical to expand the utility of cancer immunotherapy.

Efforts are underway to characterize how the crosstalk between the innate and adaptive immune response influences sustained antigen-specific immunity. ${ }^{4}$ For example, myeloid cell-targeting agents may increase the number of responders to T-cell immune checkpoint inhibitors. ${ }^{5}$ As forms of myeloid cells, macrophages and dendritic cells (DC) are the focus of a number of developmental approaches ${ }^{6-10}$ and several agents, which impact these innate cells have been recently reviewed. ${ }^{11}$

PG545 (pixatimod, INN) is a small-molecule immunomodulatory agent with strong anti-heparanase activity ${ }^{12-14}$ that has been demonstrated to potently inhibit tumour-associated macrophages (TAM) in preclinical cancer models. ${ }^{15,16}$ Because heparanase plays a key role in the molecular decision-making that guides the cancer-promoting actions of TAM in pancreatic carcinoma, ${ }^{17}$ inhibition of this protein may be responsible for the inhibition of TAM in those studies. However, PG545 also exerts an immunostimulatory effect on DC, leading to the activation of natural killer (NK) cells capable of eradicating established lymphoma in mice. ${ }^{18}$ This effect was dependent on the presence

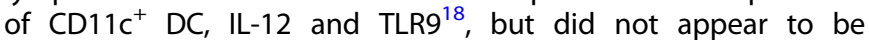
dependent on heparanase (unpublished data). Thus, PG545 modulates myeloid cells and represents a novel approach to enhance innate immunity against tumours.

As PG545 has shown significant activity in multiple tumour models, including pancreatic, colon, ovarian, breast and lung, ${ }^{13,16,19-23}$ the compound advanced into clinical testing for patients with advanced solid malignancies. Here, we report on the safety and activity of PG545 monotherapy in advanced cancer patients (NCT02042781).

\footnotetext{
${ }^{1}$ Zucero Therapeutics, Brisbane, QLD, Australia; ${ }^{2}$ Department of Surgery, Duke University Medical Center, Durham, NC, USA; ${ }^{3}$ Nucleus Network, Melbourne, VIC, Australia; ${ }^{4}$ Progen Pharmaceuticals Ltd, Brisbane, QLD, Australia; ${ }^{5}$ Clinical Network Services, Brisbane, QLD, Australia; ${ }^{6}$ Departments of Medicine and Immunology, Duke University Medical Center, Durham, NC, USA; ${ }^{7}$ Cancer Clinical Trials Unit, Royal Adelaide Hospital; Centre for Cancer Biology, SA Pathology and University of South Australia; Discipline of Medicine,

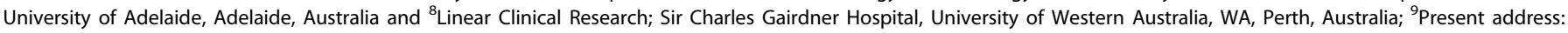
School of Humanities and Social Science, The University of Newcastle, Newcastle, NSW, Australia

Correspondence: Keith Dredge (keith.dredge@zucero.com.au)

Michael P. Brown and Michael Millward are Co-senior authors.
}

Received: 3 October 2017 Revised: 4 January 2018 Accepted: 5 January 2018

Published online: 13 March 2018 


\section{MATERIALS AND METHODS}

Patients

Twenty-three adult patients with advanced, metastatic disease that had relapsed or was refractory to standard therapy or for which no effective standard therapy was available were enrolled across three sites in Australia. Additionally, patients had to have a performance status (Eastern Cooperative Oncology Group [ECOG]) $\leq 1$ and adequate haematological, renal, cardiac and hepatic functions. All patients provided written informed consent. The study was approved by local human research ethics committees (HREC), the Royal Adelaide Hospital Research Ethics Committee (for RAH site) and Bellberry Limited (for the sites at Linear and Nucleus Network). The trial was conducted in accordance with the ICH Good Clinical Practice (GCP) guidelines and the Declaration of Helsinki.

Study design and treatment

The study was an open-label, multicentre, phase 1 dose-escalation study using a $3+3$ design. The primary objective was to determine the MTD of weekly administered PG545 via IV infusion. Secondary objectives were to characterize safety, tolerability, clinical activity, PK and PD biomarkers.

The initial cohort received 25 mg PG545 once weekly by 1-h IV infusion with dose escalation in subsequent cohorts following a pre-defined dose-escalation scheme: $50 \mathrm{mg}, 100 \mathrm{mg}$ and $150 \mathrm{mg}$. Treatment cycles were 28 days duration, with dosing occurring on days 1, 8, 15 and 22. Patients continued treatment until disease progression, unacceptable toxicity or withdrawal (either voluntarily or investigator-decision).

Safety and efficacy assessments

All patients were assessed for safety, those who received at least one dose of PG545 were evaluated for efficacy. Safety assessment included the incidence of all adverse events (AEs), irrespective of relationship to study drug, according to the National Cancer Institute Common Terminology Criteria for Adverse Events, version 4.0, and the incidence of patients experiencing dose modifications and/or premature discontinuation of study drug. Dose-limiting toxicities (DLTs) used to determine the MTD were defined as occurring during Cycle 1 of treatment only and related to PG545 according to investigator assessment. If no DLT occurred in the first three subjects, the next three-subject cohort was enrolled at the next highest available dose level. If a single DLT occurred, an additional three subjects was enrolled at the same dose level. Dose escalation continued until two subjects of the cohort experienced a DLT; this was defined as the toxic dose level. The dose-escalation phase was completed and MTD determined by reducing the dose to the next lowest dose compared to the toxic dose and expanding this cohort to at least 6 subjects.

Subjects with measurable disease were assessed by computed tomography at baseline and every 8 weeks according to Response Evaluation Criteria in Solid Tumours (RECIST), version 1.1 criteria.

Pharmacokinetics assessment

Plasma samples were obtained for the first (day 1) and fourth (day 22) doses, as follows: (1) pre-dosing (no more than $4 \mathrm{~h}$ prior to infusion start); (2) mid-point of infusion; prior to completion of infusion; 30 min post-infusion; then 2, 4, 6, 24, 48, 72, 144 and $168 \mathrm{~h}$ post-infusion. Samples were analysed for PG545 concentration using a validated liquid chromatography with tandem mass spectrometry (LC-MS/MS) assay. ${ }^{13}$ A population PK model was developed using NONMEM and individual noncompartmental analysis-type exposure parameter estimation was also performed.

Pharmacodynamics assessment

Plasma and peripheral blood mononuclear cell (PBMC) samples were obtained at various points for PD assessment with the

\begin{tabular}{|c|c|c|c|c|c|}
\hline Parameter & $\begin{array}{l}\text { Overall } \\
N=23\end{array}$ & $\begin{array}{l}25 \mathrm{mg} \\
N=3\end{array}$ & $\begin{array}{l}50 \mathrm{mg} \\
N=5\end{array}$ & $\begin{array}{l}100 \mathrm{mg} \\
N=6\end{array}$ & $\begin{array}{l}150 \mathrm{mg} \\
N=9\end{array}$ \\
\hline $\begin{array}{l}\text { Median age, years } \\
\text { (range) }\end{array}$ & $\begin{array}{l}62.0(28, \\
76)\end{array}$ & $\begin{array}{l}71.0 \\
(38,72)\end{array}$ & $\begin{array}{l}68.0 \\
(55,76)\end{array}$ & $\begin{array}{l}60.5 \\
(28,76)\end{array}$ & $\begin{array}{l}60.0 \\
(45,75)\end{array}$ \\
\hline Male, \% & 61 & 33 & 40 & 67 & 78 \\
\hline \multicolumn{6}{|l|}{ Race, $n$ (\%) } \\
\hline Caucasian & $19(83)$ & $2(67)$ & $5(100)$ & $5(83)$ & $7(78)$ \\
\hline Asian & $2(9)$ & 0 & 0 & $1(17)$ & $1(11)$ \\
\hline Other & $2(9)$ & $1(33)$ & 0 & 0 & $1(11)$ \\
\hline \multicolumn{6}{|l|}{ ECOG, $n(\%)$} \\
\hline 0 & $13(57)$ & $3(100)$ & $4(80)$ & $2(33)$ & $4(44)$ \\
\hline 1 & $10(43)$ & 0 & $1(20)$ & $4(67)$ & $5(56)$ \\
\hline \multicolumn{6}{|c|}{ Stage of disease, $n(\%)$} \\
\hline IIIC & $1(4)$ & 0 & $1(20)$ & 0 & 0 \\
\hline IV & $19(83)$ & $2(67)$ & $4(80)$ & $5(83)$ & $8(89)$ \\
\hline Unknown & $2(9)$ & $1(33)$ & 0 & $1(17)$ & 0 \\
\hline N/A & $1(4)$ & 0 & 0 & 0 & $1(11)$ \\
\hline \multicolumn{6}{|l|}{ Prior therapy, $n$ (\%) } \\
\hline Chemotherapy & $18(78)$ & 0 & $4(80)$ & $6(100)$ & $8(89)$ \\
\hline Surgery & $17(74)$ & $3(100)$ & $4(80)$ & $4(67)$ & $6(67)$ \\
\hline Radiotherapy & $11(48)$ & $2(67)$ & $2(40)$ & $3(50)$ & $4(44)$ \\
\hline Other & $13(57)$ & $2(67)$ & $2(40)$ & $5(83)$ & $4(44)$ \\
\hline
\end{tabular}

exception of the $25 \mathrm{mg}$ cohort, where PBMC were not collected. Plasma samples were analysed for the concentration of various markers, including cytokines, chemokines and heparanase using commercial ELISA (LifeSpan Biosciences, Seattle, WA), and array kits (Quansys, Logan, UT).

Cryopreserved PBMC samples were analysed by flow cytometry for changes in abundance and activation status of DC and NK cells at day 3 post-treatment compared with pre-dose samples (day 1). Given the stimulatory nature of PG545 via TLR9 reported in mice, ${ }^{18}$ efforts were primarily focussed on the assessments of $\mathrm{pDC}$ and NK cells though certain subjects with available sample underwent additional analyses. To assess $\mathrm{pDC}$, cells were stained with antibodies to HLADR-peCy7, CD303-FITC, IFNa-647 (BD Biosciences, Franklin Lakes, NJ). To assess NK cells, cells were stained with antibodies to CD335-PE, CD3-APC, IFNY-FITC (BD Biosciences). All intracellular cytokine staining samples were stimulated by 100 $\mathrm{ng} / \mathrm{mL}$ PMA, $100 \mathrm{ng} / \mathrm{mL}$ ionomycin and $5 \mu \mathrm{g} / \mathrm{mL}$ Brefeldin A for $4 \mathrm{~h}$ at $37{ }^{\circ} \mathrm{C}$. Samples were acquired using FACSCanto flow cytometers (BD Biosciences).

\section{RESULTS}

Patient demographics and baseline characteristics

Twenty-three patients were screened, enrolled, and received at least one dose of PG545 during the study. Baseline demographic and clinical characteristics of the population are summarised in Table 1.

\section{Safety}

Dosing with PG545 began at $25 \mathrm{mg}$ per patient per week (3 subjects) and escalated to $50 \mathrm{mg}$ (5 subjects), $100 \mathrm{mg}$ (6 subjects) and finally $150 \mathrm{mg}$ (9 subjects). Details of enrollment and patient disposition are shown in the CONSORT diagram in the supplementary material (Supplementary Figure S1). Three DLT were observed in three patients within the $150 \mathrm{mg}$ cohort during the first cycle and this was declared the toxic dose. The DLT were two cases of hypertension (3069 with no prior history and 3063 


\begin{tabular}{|llllll}
\hline Table 2. Treatment-related AEs ( $\geq 2$ subjects) & & \\
\hline Event Type & Overall & $25 \mathrm{mg}$ & $50 \mathrm{mg}$ & $100 \mathrm{mg}$ & $150 \mathrm{mg}$ \\
& $n(\%)$ & $n(\%)$ & $n(\%)$ & $n(\%)$ & $n(\%)$ \\
& $N=23$ & $N=3$ & $N=5$ & $N=6$ & $N=9$ \\
\hline Hypertension & $6(26)$ & 0 & $1(20)$ & 0 & $5(56)$ \\
Chills & $5(22)$ & 0 & $1(20)$ & 0 & $4(44)$ \\
Fatigue & $5(22)$ & 0 & $2(40)$ & $2(33)$ & $1(11)$ \\
Pyrexia & $5(22)$ & 0 & $3(60)$ & $1(17)$ & $1(11)$ \\
Nausea & $5(22)$ & 0 & $4(80)$ & 0 & $1(11)$ \\
Decreased appetite & $5(22)$ & 0 & $2(40)$ & $1(17)$ & $2(22)$ \\
Infusion-related reaction & $5(22)$ & 0 & 0 & 0 & $5(56)$ \\
Diarrhoea & $4(17)$ & 0 & $1(20)$ & $2(33)$ & $1(11)$ \\
Hypercholesterolemia & $2(9)$ & 0 & 0 & $2(33)$ & 0 \\
Injection site reaction & $2(9)$ & $2(67)$ & 0 & 0 & 0 \\
Blood cholesterol increased & $2(9)$ & 0 & 0 & $1(17)$ & $1(11)$ \\
Headache & $2(9)$ & 0 & 0 & $2(33)$ & 0
\end{tabular}

with history of hypertension but no anti-hypertensive medication at screening) and one case of epistaxis (with prior history). Although the hypertension occurred typically during or $1 \mathrm{~h}$ postinfusion and was transient, the events were typically associated with an increase in systolic pressure to $170 \mathrm{~mm} \mathrm{Hg}$ and diastolic pressure to $100 \mathrm{~mm} \mathrm{Hg}$, with one subject experiencing a substantial increase in blood pressure (to 200/126 mm Hg). Both patients with hypertension continued on study after the DLT event, while the patient with epistaxis withdrew consent immediately following the DLT event. At that point, the $100 \mathrm{mg}$ cohort was expanded and further three patients were treated. With no further DLT observed, the $100 \mathrm{mg}$ dose was declared the MTD.

The most common treatment-related $\mathrm{AE}$ was hypertension with six occurrences, five in the $150 \mathrm{mg}$ cohort (Table 2). All treatmentemergent $A E$ are reported in supplementary material (Table S1). Of the 12 serious adverse events (SAEs), one was mild, nine were severe and two were fatal in grading (death due to disease progression). Six were assessed as possibly, likely, or certainly related to PG545 by the investigators (Table 3). Five of the SAEs resulted in patient withdrawal from the study or discontinuation of PG545 therapy. Only one patient (3069) experienced two different SAEs possibly related to PG545 (acute pulmonary oedema and severe hypertension).

Clinical activity

Sixteen patients had efficacy assessments during PG545 treatment and six of these had stable disease (SD) at 8 weeks as measured by RECIST 1.1 criteria. This ratio, six of sixteen assessed, represents a $38 \%$ disease control rate at 8 weeks (Fig. 1). The estimated median duration of SD for patients on the study was 57 days (95\% confidence interval from 39 days to 62 days). Also included in the efficacy assessment were three patients with progressive disease (3021, 2062 and 2064) who did not receive the expected 8 doses scheduled. Seven patients were censored as part of the analysis, having no recorded findings of death or progressive disease.

\section{Pharmacokinetics}

Cohort averaged data for PG545 plasma concentrations are plotted in Fig. 2a. A population pharmacokinetic model described the plasma concentrations after IV administration with good precision and little bias. PG545 had a small apparent central volume (Vc, $2.98 \mathrm{~L})$, and apparent peripheral volume $(\mathrm{Vp}, 11.9 \mathrm{~L})$
Table 3. Treatment-related severe AEs, AEs leading to discontinuation and serious AEs

\begin{tabular}{|c|c|c|c|c|c|}
\hline Event Type & $\begin{array}{l}\text { Overall } \\
n(\%) \\
N=23\end{array}$ & $\begin{array}{l}25 \mathrm{mg} \\
n(\%) \\
N=3\end{array}$ & $\begin{array}{l}50 \mathrm{mg} \\
n(\%) \\
N=5\end{array}$ & $\begin{array}{l}100 \mathrm{mg} \\
n(\%) \\
N=6\end{array}$ & $\begin{array}{l}150 \mathrm{mg} \\
n(\%) \\
N=9\end{array}$ \\
\hline \multicolumn{6}{|l|}{ Related severe AEs } \\
\hline Hypertension & $5(22)$ & 0 & 0 & 0 & $5(56)$ \\
\hline Chills & $2(9)$ & 0 & $1(20)$ & 0 & $1(11)$ \\
\hline Fatigue & $2(9)$ & 0 & $1(20)$ & $1(17)$ & 0 \\
\hline Pyrexia & $1(4)$ & 0 & $1(20)$ & 0 & 0 \\
\hline $\begin{array}{l}\text { Blood cholesterol } \\
\text { increased }\end{array}$ & $1(4)$ & 0 & 0 & 0 & $1(11)$ \\
\hline Blood pressure increased & $1(4)$ & 0 & 0 & 0 & $1(11)$ \\
\hline $\begin{array}{l}\text { Acute pulmonary } \\
\text { oedema }\end{array}$ & $1(4)$ & 0 & 0 & 0 & $1(11)$ \\
\hline Epistaxis & $1(4)$ & 0 & 0 & 0 & $1(11)$ \\
\hline Infusion-related reaction & $1(4)$ & 0 & 0 & 0 & $1(11)$ \\
\hline Hyperlipidaemia & $1(4)$ & 0 & 0 & $1(17)$ & 0 \\
\hline \multicolumn{6}{|c|}{ Related leading to discontinuation } \\
\hline Pyrexia & $1(4)$ & 0 & $1(20)$ & 0 & 0 \\
\hline Chills & $1(4)$ & 0 & $1(20)$ & 0 & 0 \\
\hline Epistaxis & $1(4)$ & 0 & 0 & 0 & $1(11)$ \\
\hline $\begin{array}{l}\text { Acute pulmonary } \\
\text { oedema }\end{array}$ & $1(4)$ & 0 & 0 & 0 & $1(11)$ \\
\hline Hypertension & $1(4)$ & 0 & 0 & 0 & $1(11)$ \\
\hline \multicolumn{6}{|l|}{ Related serious $\mathrm{AE}$} \\
\hline $\begin{array}{l}\text { Acute pulmonary } \\
\text { oedema }\end{array}$ & $1(4)$ & 0 & 0 & 0 & $1(11)$ \\
\hline Epistaxis & $1(4)$ & 0 & 0 & 0 & $1(11)$ \\
\hline Pyrexia & $1(4)$ & 0 & $1(20)$ & 0 & 0 \\
\hline Infusion-related reaction & $1(4)$ & 0 & 0 & 0 & $1(11)$ \\
\hline Hypovolaemia & $1(4)$ & 0 & 0 & $1(17)$ & 0 \\
\hline Hypertension & $1(4)$ & 0 & 0 & 0 & $1(11)$ \\
\hline
\end{tabular}

and a slow apparent clearance $(\mathrm{CL}, 0.0165 \mathrm{~L} / \mathrm{h})$, resulting in a long mean $t_{1 / 2}$ estimated as $141 \mathrm{~h}$ across all subjects. Between subject variability was large for apparent peripheral volume (Vp, $61.2 \% \mathrm{CV})$ and $\mathrm{CL}(75.6 \% \mathrm{CV})$. The model and individual estimates for exposure (such as AUC and $C_{\max }$ ) indicated that the exposure was not dose proportional with individual exposure for the $150 \mathrm{mg}$ dosing group, which was being less than that predicted from lower-dosing groups if dose-proportionality was assumed. Individual estimates of area under the curve over the dosing interval $\left(A \cup C_{0-\text { last }}\right)$ after first dose indicated a general dose-proportionate exposure up to $100 \mathrm{mg}$ (Fig. $2 \mathrm{~b}$ and Table A1). Maximum observed PG545 concentration, derived from the measured concentration values $\left(C_{\max }\right)$ was also broadly proportional up to $100 \mathrm{mg}$ (Fig. 2c and Table S2).

Pharmacodynamics

Analysis of patient PBMC 2 days after the initial PG545 dose, revealed that, at the 50 and $100 \mathrm{mg}$ dose levels, six of the ten subjects analysed in these cohorts exhibited at least two-fold increased numbers of either, or both, activated pDC or NK cells (Fig. 3). Two subjects had greater than two-fold increases in $\mathrm{CD} 40^{+}$ pDC (3024 and 1045) with the same two and subject 1041 showing increases in IFNa expressing pDC (Fig. 3a,b). Three subjects, including two showing DC activation (3024 and 1041), and 3043 had increased numbers of the NKp $46^{+}$NK cells (Fig. 3c). There was 


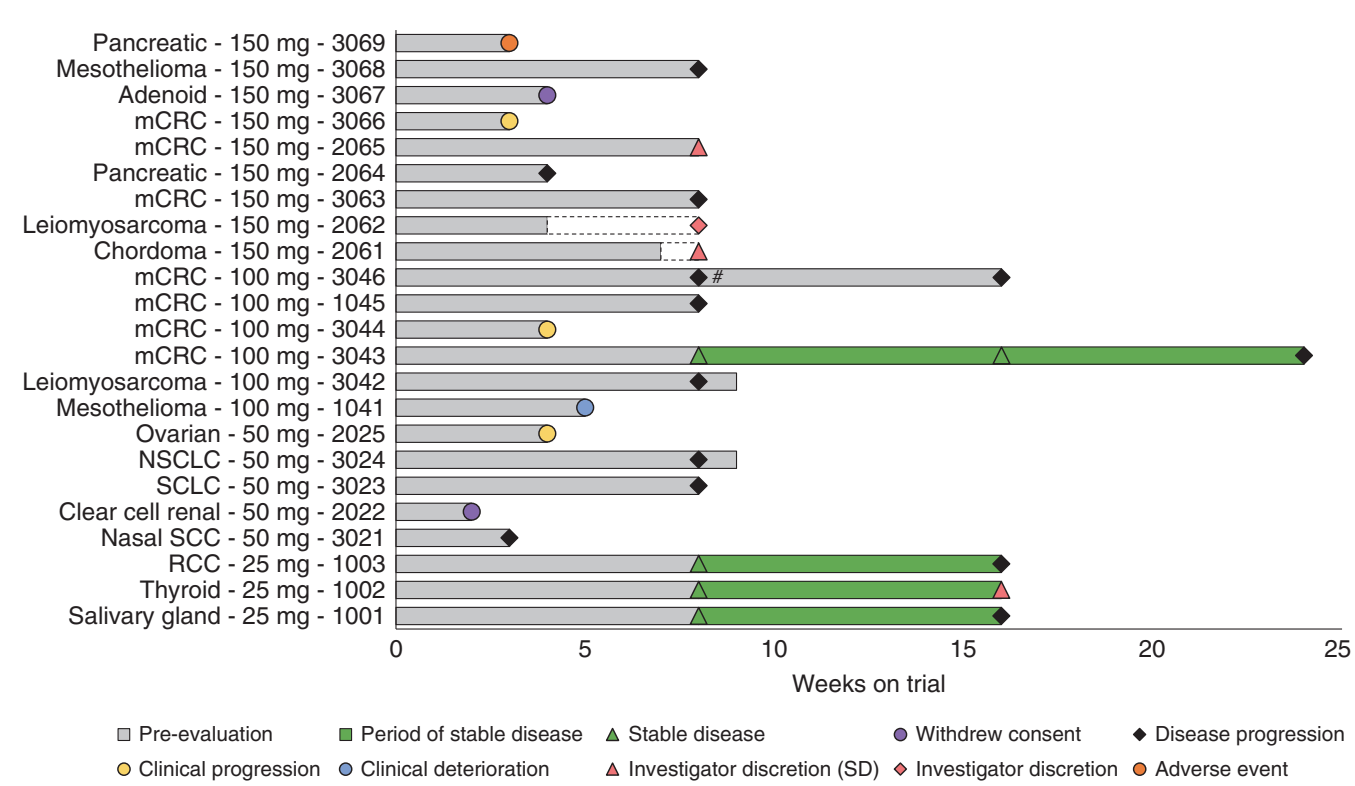

Fig. 1 PG545 clinical activity. Cancer type, dose and subject ID indicated for the 23 subjects on trial and weeks on trial are shown in the swim plot. Dashed bars (subjects 2061 and 2062) indicate that subjects stopped receiving PG545 due to investigator discretion but stayed on study until 8 week assessment. " Subject stayed on study after PD erroneously classified as SD at 8 week. mCRC metastatic colorectal cancer, NSCLC non-small cell lung cancer, SCLC small cell lung cancer, SCC squamous cell carcinoma, RCC renal cell carcinoma
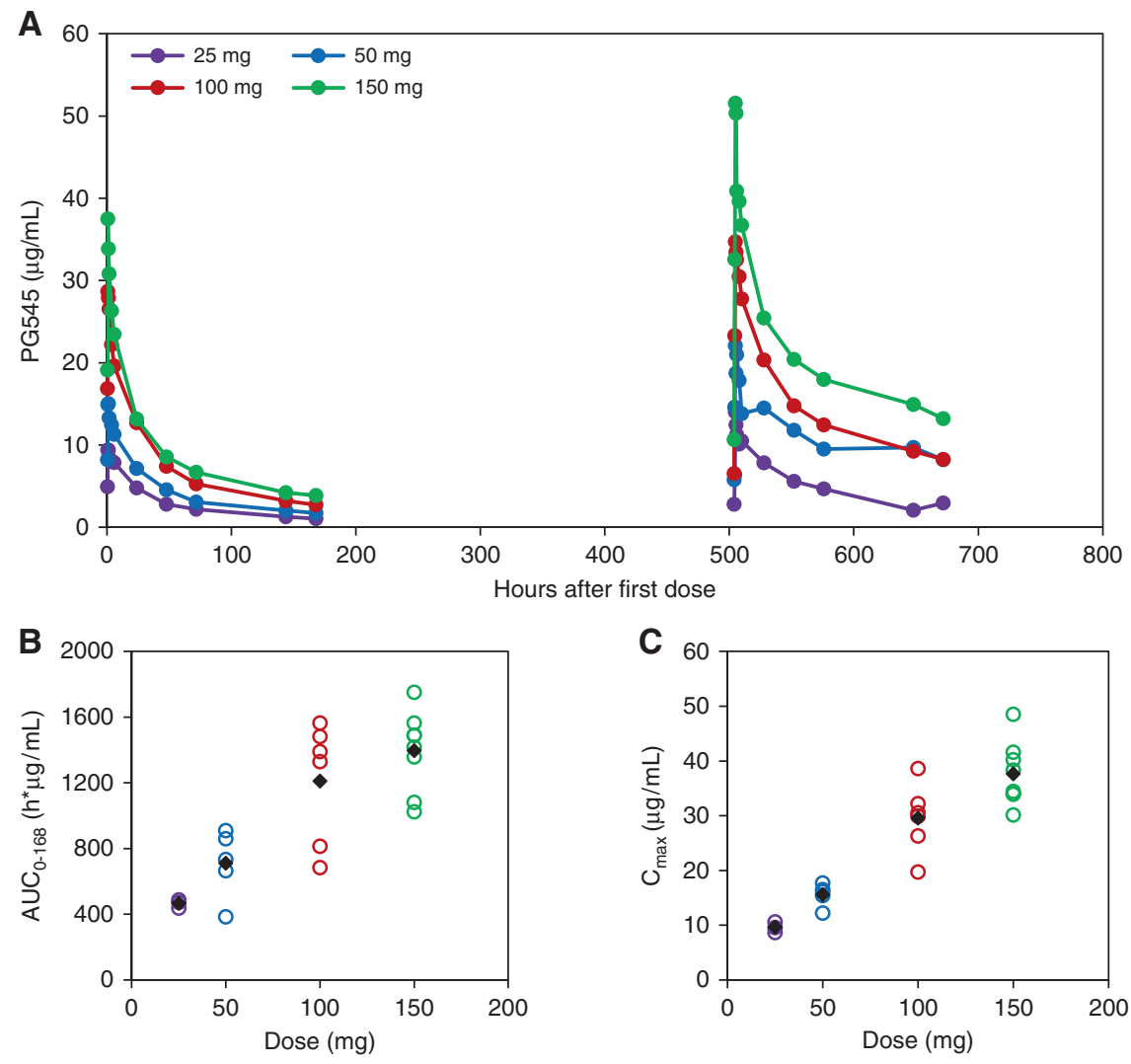

Fig. 2 PG545 pharmacokinetics. Mean plasma PG545 concentration vs. time curves following first and fourth dose (a). Dose-proportionality plots for $A U C_{0-168}$ (b) and $C_{\max }$ (c) yields a proportional exposure response to dosing, up to $100 \mathrm{mg}$. At the $150 \mathrm{mg}$ dose level, exposure appears to be sub-proportional for $A U C_{0-168}$ and $C_{\max }$ values. In $\mathbf{b}, \mathbf{c}$, the data for individual subjects are represented by open circles and cohort averages are represented as black diamonds

also evidence of activation of NK cells, as indicated by increased IFNY expression, in three subjects (Fig. 3d). In the $150 \mathrm{mg}$ cohort, these parameters were unaffected or modestly responsive following treatment, though some subjects had increased expression of CD40 on conventional DCs (Supplementary Figure S2). PBMC were not collected for the $25 \mathrm{mg}$ cohort.

Concentrations of selected signalling proteins were measured in subject plasma before and after the initial PG545 dose. Increases, 
A 7

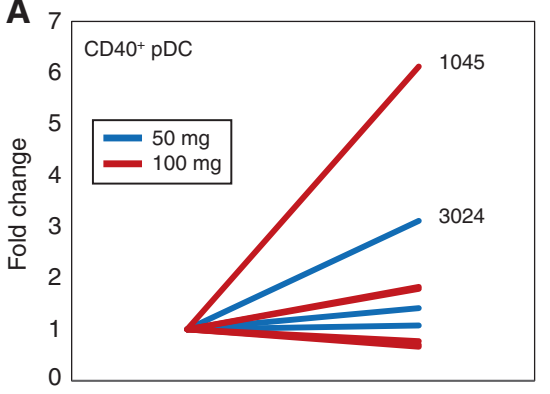

B

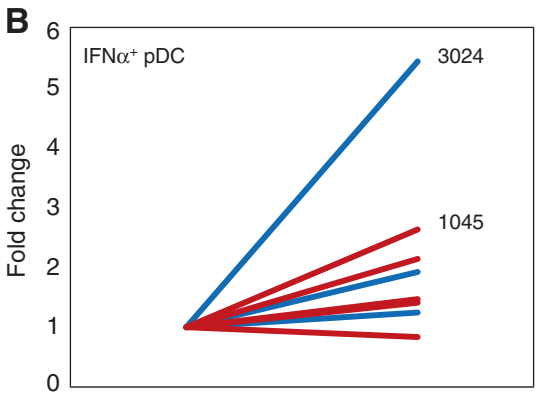

C
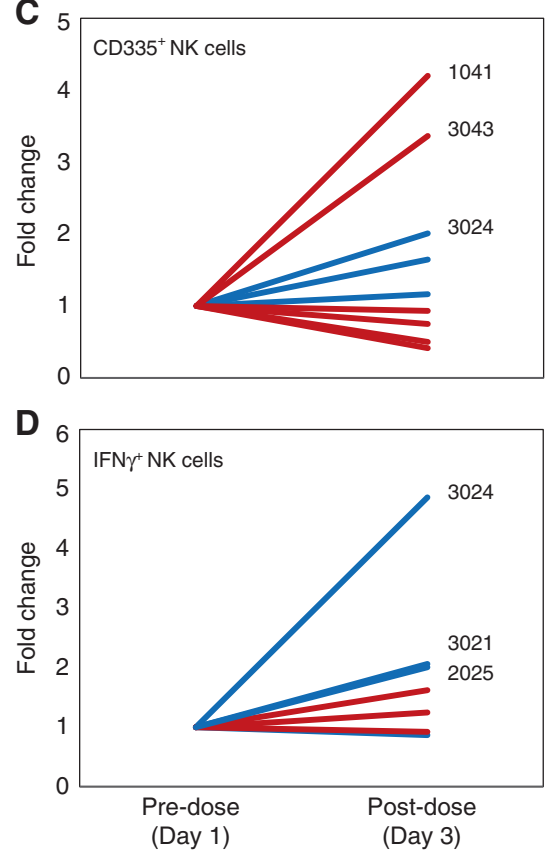
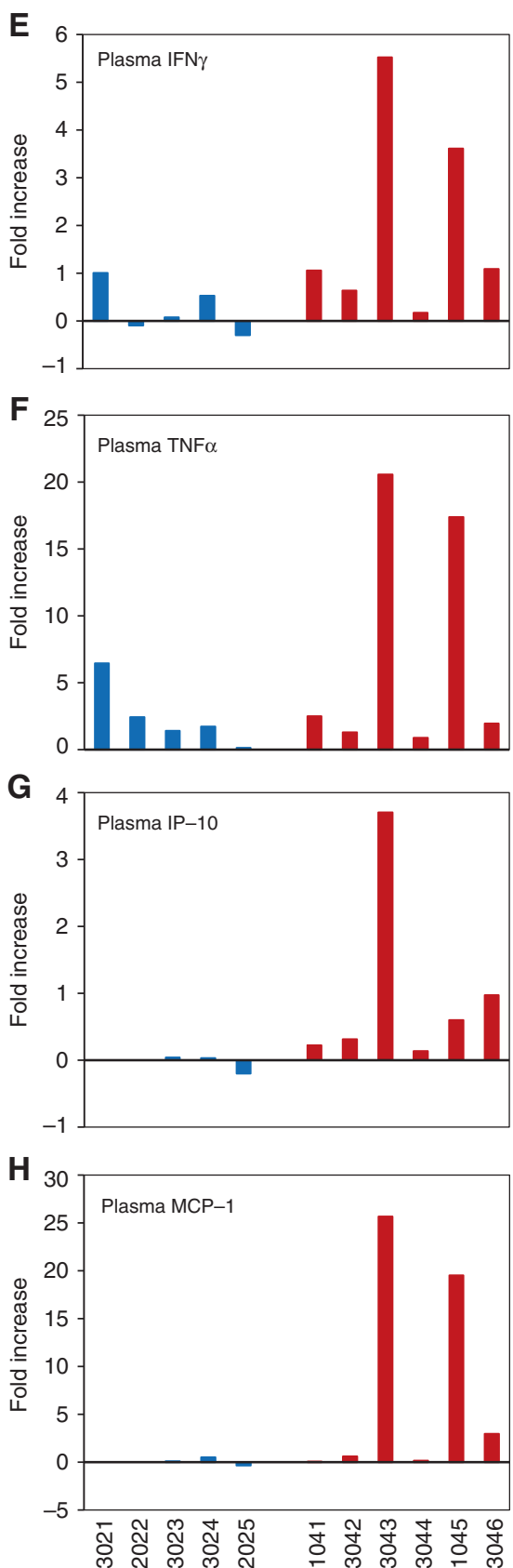

Fig. 3 PG545 pharmacodynamics. Flow cytometry analysis of PBMC from the 50 and $100 \mathrm{mg}$ cohorts showing increased expression of CD40 (a) and IFN $\alpha$ by pDC (b) and increased expression of NKp46 (c) and IFN $\gamma$ by NK cells (d) after PG545 treatment. Patient numbers are indicated where increases are greater than two-fold. Analysis of plasma biomarkers shows increases in IFN $\gamma(\mathbf{e})$, TNF $\alpha$ (f), IP-10 (g) and MCP-1 (h) after PG545 treatment in the $50 \mathrm{mg}$ and $100 \mathrm{mg}$ cohorts

of up to 25-fold, were found in plasma IFNy, TNFa, IP-10 (CXCL10) and MCP-1 (CCL2) following PG545 treatment (Fig. 3e-h). Though cohort averages reveal PG545-induced elevations in IFNy were similar between $100 \mathrm{mg}$ and $150 \mathrm{mg}$ cohorts, increases in TNFa, IL6 and IL-8 responses were strikingly higher at $150 \mathrm{mg}$ (Supplementary Figure S3). Changes in plasma concentrations for a range of cytokines, chemokines and potential pharmacodynamic markers are shown in the appendix (Supplementary Figures S3 and S4).

A summary of the immune modulation status following the first PG545 dose is provided in a matrix (Supplementary Table S3).

\section{DISCUSSION}

PG545 is a novel immunomodulatory agent, which has been demonstrated to possess potent anti-tumour activity in multiple preclinical models of cancer. ${ }^{12-16,18-23}$ Herein, we report on the first Phase 1 study of PG545 as an IV infusion in subjects with advanced cancer.

Treatment with PG545 was well tolerated, though paracetamol was required at doses above the $25 \mathrm{mg}$ cohort to abrogate reactions associated with the infusion such as pyrexia and chills. Given that the immunostimulatory property of PG545 appears to be mediated via a TLR9-dependent mechanism, ${ }^{18}$ the flu-like symptoms and infusion reactions appear consistent with AEs 
associated with TLR9 agonists. ${ }^{24}$ However, the protective effect of paracetamol was lost once the dose reached $150 \mathrm{mg}$ and additional pre-medications (cetirizine, amlodipine and hydrocortisone) had varying, but ultimately limited impact, on an emerging $\mathrm{AE}$ of acute transient hypertension during or shortly following infusion of PG545. Given the rapid onset and transient nature $(<24 \mathrm{~h})$ of the hypertensive episodes associated with PG545, it is unlikely that the effect is mediated through a similar mechanism as that described for anti-VEGF therapies. ${ }^{25}$ However, it is possible that activation of TLR9 could lead to hypertension. ${ }^{26}$ The elevation of plasma IL-8 levels in the $150 \mathrm{mg}$ cohort could indicate that endothelial cells, which can express TLR9 and respond to TLR9 agonists, may be stimulated by this highest dose to release IL-8, a cytokine that can contribute to hypertension. ${ }^{27,28}$ Hypertension was not observed in the $100 \mathrm{mg}$ cohort.

The lack of toxicity associated with the MTD was encouraging and the exposure achieved in these subjects (based on $A U C_{0-l a s t}$ values) was higher compared with mice receiving efficacious doses of PG545. ${ }^{13,22}$ Moreover, the AUC was sub-proportional for the $150 \mathrm{mg}$ dose level. Simulation data using the model indicated that there were prolonged exposure and some accumulation after multiple weekly doses. Total exposure was reduced when simulation of a reduced dosing frequency after 4 weekly doses to once every 2 weeks was modelled. However, as the population mean minimum concentration over the dosing interval, based on this simulation, after 16 weeks (4 cycles) was approximately half $(10 \mu \mathrm{g} / \mathrm{mL}$ versus $21 \mu \mathrm{g} / \mathrm{mL})$ of that observed after once-weekly dosing at $100 \mathrm{mg}$ (Supplementary Figure S5), any future modifications of dosing frequency could only be considered once exposure and its relationship to clinical activity are apparent.

The lack of objective responses using PG545 as a monotherapy in this clinical study is somewhat consistent with the published preclinical data in advanced disease settings, namely that only PG545 in combination with chemotherapy can eradicate large established tumours. ${ }^{18}$ Given the tremendous potential for combination approaches to extend the clinical success of immunotherapies ${ }^{29}$ and that maximal immunity is achieved only when both the innate and adaptive arms of the immune system work in concert, ${ }^{30}$ PG545 could also enhance clinical responses to T-cell-targeting immunotherapy such as immune checkpoint inhibitors by targeting innate immune cells. To that end, a new Phase $1 \mathrm{~b}$ trial for metastatic pancreatic cancer is assessing the safety and tolerability of escalating doses of PG545 (25, 50 and $100 \mathrm{mg}$ ) with the anti-PD1 drug nivolumab (ANZCTR trial ID ACTRN12617001573347), which could further characterise the biologically optimal dose of PG545.

Preliminary pharmacodynamic analyses of PG545 identified changes in cell surface markers, cytokines and chemokines, which is consistent with preclinical findings ${ }^{18}$ and other innate stimulatory agents. ${ }^{31-35}$ At 50 and $100 \mathrm{mg}$ dose levels, activation in pDCs of some patients was apparent either by elevations in CD40 expression or IFNa production, both of which are considered key targets for the treatment of cancer, $6,10,36,37$ while activation of NK cells was evident either by increased numbers of NKp46 ${ }^{+}(\mathrm{CD} 335)$ cells or IFNy production. Together with increases in several plasma cytokines and chemokines noted within $24 \mathrm{~h}$ of first PG545 treatment, for example, IFN $\gamma$, MCP-1 and IP-10 in the $100 \mathrm{mg}$ cohort, such changes have been previously associated with immunomodulators such as TLR agonists. ${ }^{31-35}$ Though these changes may have some utility as biomarkers of inflammation and immune function, the new clinical study will examine not only T-cell-associated markers in PBMCs but also immune cell populations within the tumour microenvironment (TME), as this may be more predictive of clinical responses.
In conclusion, the study demonstrated that PG545 is well tolerated in patients with advanced solid malignancies and treatment was associated with changes in proposed pharmacodynamic markers. By modulating myeloid cells in the TME-as demonstrated in preclinical models via stimulation of DC to drive IL-12/TLR9/NK cell-dependent destruction of lymphoma in mice ${ }^{18}$ and inhibition of M2 macrophages and MDSCs ${ }^{15,16}$ - PG545 offers a complementary approach for use in combination with existing therapies.

\section{ACKNOWLEDGEMENTS}

We thank the patients and their families who participated in this study, and the study teams at Linear Clinical Research (Perth), Nucleus Network (Melbourne) and the Royal Adelaide Hospital (Adelaide). They also thank the personnel at TetraQ (The University of Queensland, Brisbane) for the bioanalysis of PG545 and Dalia Khalil, David Sester and Kristen Radford at the Translational Research Institute (Brisbane) for their technical assistance with flow cytometry. The clinical trial described in this work was sponsored and supported by Progen Pharmaceuticals Ltd (now TBG Diagnostics) and Zucero Therapeutics Ltd.

\section{ADDITIONAL INFORMATION}

Supplementary information is available for this paper at https://doi.org/10.1038/ s41416-018-0006-0.

Competing interests: K.D., E.H., F.L., D.B., P.H. were or are employees or consultants of Progen Pharmaceuticals Ltd or Zucero Therapeutics Ltd. The remaining authors declare that they have no competing of interest.

Note: This work is published under the standard license to publish agreement. After 12 months the work will become freely available and the license terms will switch to a Creative Commons Attribution 4.0 International licence (CC BY 4.0).

\section{REFERENCES}

1. Postow, MA., Callahan, MK. \& Wolchok, JD. Immune checkpoint blockade in cancer therapy. J Clin Oncol 17, 1974-1982 (2015).

2. Kreamer, K. M. Immune checkpoint blockade: a new paradigm in treating advanced cancer. J. Adv. Pract. Oncol. 5, 418-431 (2014).

3. Zhao, X. \& Subramanian, S. Intrinsic resistance of solid tumors to immune checkpoint blockade therapy. Cancer Res. 77, 817-822 (2017).

4. Sayour, E. J. \& Mitchell, D. A. Manipulation of innate and adaptive immunity through cancer vaccines. J. Immunol. Res 2017, 3145742 (2017).

5. Engblom, C., Pfirschke, C. \& Pittet, M. J. The role of myeloid cells in cancer therapies. Nat. Rev. Cancer 16, 447-462 (2016).

6. Beatty, G. L., Li, Y. \& Long, K. B. Cancer immunotherapy: activating innate and adaptive immunity through CD40 agonists. Expert. Rev. Anticancer. Ther. 17, 175-186 (2017).

7. Mitchem, J. B. et al Targeting tumor-infiltrating macrophages decreases tumorinitiating cells, relieves immunosuppression, and improves chemotherapeutic responses. Cancer Res. 73, 1128-1141 (2013).

8. Morrison, C. Immuno-oncologists eye up macrophage targets. Nat. Rev. Drug. Discov. 15, 373-374 (2016).

9. Zhu, Y. et al CSF1/CSF1R blockade reprograms tumor-infiltrating macrophages and improves response to T-cell checkpoint immunotherapy in pancreatic cancer models. Cancer Res. 74, 5057-5069 (2014).

10. Zitvogel, L., Galluzzi, L., Kepp, O., Smyth, M. J. \& Kroemer, G. Type I interferons in anticancer immunity. Nat. Rev. Immunol. 15, 405-414 (2015).

11. Smyth, M. J., Ngiow, S. F., Ribas, A. \& Teng, M. W. L. Combination cancer immunotherapies tailored to the tumour microenvironment. Nat. Rev. Clin. Oncol. 13, 143-158 (2016).

12. Dredge, K. et al The PG500 series: novel heparan sulfate mimetics as potent angiogenesis and heparanase inhibitors for cancer therapy. Invest. New Drugs $\mathbf{2 8 ,}$ 276-283 (2010).

13. Dredge, K. et al PG545, a dual heparanase and angiogenesis inhibitor, induces potent anti-tumour and anti-metastatic efficacy in preclinical models. Br. J. Cancer 104, 635-642 (2011).

14. Ferro, V. et al Discovery of PG545: a highly potent and simultaneous inhibitor of angiogenesis, tumor growth, and metastasis. J. Med. Chem. 55, 3804-3813 (2012).

15. Boyango, I. et al Heparanase cooperates with Ras to drive breast and skin tumorigenesis. Cancer Res. 74, 4504-4514 (2014). 
16. Ostapoff, K. T. et al PG545, an angiogenesis and heparanase inhibitor, reduces primary tumor growth and metastasis in experimental pancreatic cancer. Mol. Cancer Ther. 12, 1190-1201 (2013).

17. Hermano $E$, et al. Macrophage polarization in pancreatic carcinoma: role of heparanase enzyme. J. Natl Cancer. Inst. 106, dju332 (2014).

18. Brennan, T. V. et al Heparan sulfate mimetic PG545-mediated antilymphoma effects require TLR9-dependent NK cell activation. J. Clin. Invest. 126, 207-219 (2016).

19. Jung, D.-B. et al The heparan sulfate mimetic PG545 interferes with Wnt/ 3 catenin signaling and significantly suppresses pancreatic tumorigenesis alone and in combination with gemcitabine. Oncotarget 6, 4992-5004 (2015).

20. Mondal, S. et al HSulf-1 deficiency dictates a metabolic reprograming of glycolysis and TCA cycle in ovarian cancer. Oncotarget 6, 33705-33719 (2015).

21. Singh, P. et al The heparanase inhibitor PG545 attenuates colon cancer initiation and growth, associating with increased p21 expression. Neoplasia 19, 175-184 (2017).

22. Winterhoff, B. et al PG545 enhances anti-cancer activity of chemotherapy in ovarian models and increases surrogate biomarkers such as VEGF in preclinical and clinical plasma samples. Eur. J. Cancer 51, 879-892 (2015).

23. Hammond, E., Brandt, R. \& Dredge, K. PG545, a heparan sulfate mimetic, reduces heparanase expression in vivo, blocks spontaneous metastases and enhances overall survival in the 4T1 breast carcinoma model. PLOS ONE 7, e52175 (2012).

24. Krieg, A. M. Toll-like receptor 9 (TLR9) agonists in the treatment of cancer. Oncogene 27, 161-167 (2008). 10.1038/sj.onc.1210911.

25. Hayman, S. R., Leung, N., Grande, J. P. \& Garovic, V. D. VEGF inhibition, hypertension, and renal toxicity. Curr. Oncol. Rep. 14, 285-294 (2012).

26. McCarthy, C. G. et al Circulating mitochondrial DNA and Toll-like receptor 9 are associated with vascular dysfunction in spontaneously hypertensive rats. Cardiovasc. Res. 107, 119-130 (2015).
27. Kim, H. Y. et al. Reparixin, an inhibitor of CXCR1 and CXCR2 receptor activation, attenuates blood pressure and hypertension-related mediators expression in spontaneously hypertensive rats. Biol. Pharm. Bull. 34, 120-127 (2011).

28. Li, J. et al CpG DNA-mediated immune response in pulmonary endothelial cells. Am. J. Physiol. Lung Cell. Mol. Physiol. 287, L552-L558 (2004).

29. Ott, P. A., Hodi, F. S., Kaufman, H. L., Wigginton, J. M. \& Wolchok, J. D. Combination immunotherapy: a road map. J. Immunother. Cancer 5, 16 (2017).

30. Shalapour, S. \& Karin, M. Immunity, inflammation, and cancer: an eternal fight between good and evil. J. Clin. Invest. 125, 3347-3355 (2015).

31. Molenkamp, B. G. et al Intradermal CpG-B activates both plasmacytoid and myeloid dendritic cells in the sentinel lymph node of melanoma patients. Clin. Cancer Res. 13, 2961-2969 (2007).

32. Northfelt, D. W. et al A phase I dose-finding study of the novel Toll-like receptor 8 agonist VTX-2337 in adult subjects with advanced solid tumors or lymphoma. Clin. Cancer Res. 20, 3683-3691 (2014).

33. Weber, J. S. et al Randomized phase $2 / 3$ trial of CpG oligodeoxynucleotide PF3512676 alone or with dacarbazine for patients with unresectable stage III and IV melanoma. Cancer 115, 3944-3954 (2009).

34. Weihrauch, M. R. et al Phase I clinical study of the toll-like receptor 9 agonist MGN1703 in patients with metastatic solid tumours. Eur. J. Cancer 51, 146-156 (2015)

35. Wittig, B., Schmidt, M., Scheithauer, W. \& Schmoll, H.-J. MGN1703, an immunomodulator and toll-like receptor 9 (TLR-9) agonist: from bench to bedside. Crit. Rev. Oncol. Hematol. 94, 31-44 (2015).

36. Johnson, P. et al Clinical and biological effects of an agonist anti-CD40 antibody: a Cancer Research UK phase I study. Clin. Cancer Res. 21, 1321-1328 (2015).

37. Zoglmeier, C. et al CpG blocks immunosuppression by myeloid-derived suppressor cells in tumor-bearing mice. Clin. Cancer Res. 17, 1765-1775 (2011). 